\title{
Étude de la dégradation de deux boissons industrielles consommées en mairie de Bujumbura, Burundi.
}

Jean Chrysostome Ndamanisha, Pierre Claver Habonimana et Gordien Ntahimpera

Université du Burundi, Département de Chimie, B. P. 1550, 2 Avenue de l'UNESCO, Bujumbura- Burundi

Corresponding author email: jean-chrysostome.ndamanisha@ub.edu.bi

Original submitted in on $1^{\text {st }}$ February 2017. Published online at www.m.elewa.org on $31^{\text {st }}$ May 2017 https://dx.doi.org/10.4314/jab.v113i1.9

\section{RESUME}

Objectif : Des fois, les consommateurs des bières disent que le produit vendu a perdu le goût de la bière et ils pensent qu'elle a été dégradée. Les vendeurs quant à eux, exhibent la date d'expiration marquée sur la bouteille pour montrer que la bière est encore en bon état. L'objectif de ce travail est d'essayer de résoudre cet état d'incompréhension entre l'acheteur et le vendeur de cette bière.

Méthodologie et Résultats : Deux bières consommées au Burundi (Amstel beer et Amstel bock) ont été conservées à l'air libre dans un magasin, en la mairie de Bujumbura. Les paramètres physico- chimiques de ces bières ont été analysés pendant six mois de conservation, date d'expiration indiquée sur la bouteille d'emballage. Les résultats montrent que les paramètres étudiés ( $\mathrm{pH}$, la teneur en alcool, l'acidité totale titrable, et l'acidité volatile) varient au cours du temps de conservation. Cependant, pendant les six mois de conservation, ces paramètres restent dans les limites établies pour une bière.

Conclusion et Application des résultats : Le goût de la bière peut effectivement changer au cours du temps de conservation, mais la bière est considérée encore en bon état avant les six mois de conservation. Cependant " ce bon état » est quand même différent de l'état correspondant au temps $t=0$ de conservation. Le résultat de ce travail peut aider à gérer certains conflits entre l'acheteur et le vendeur de ces deux bières.

Mots-clés : Amstel beer, Amstel bock, paramètres physico-chimiques, goût, dégradation, conservation, Bujumbura.

\footnotetext{
ABSTRACT

Study of the degradation of two industrial drinks consumed in Bujumbura city, Burundi.Objective: Objective: Consumers of beer, sometimes, say that the product has lost the taste of beer and they think that it has been degraded. The sellers, meanwhile, mark the expiry date on the bottle to show that the beer is still in good condition. The purpose of this work is to try to resolve this lack of understanding between the buyer and the seller of this beer.

Methodology and Results: Two beers consumed in Burundi (Amstel beer and Amstel bock) were stocked in a store at air dry, in Bujumbura city. The physicochemical parameters of these beers were analyzed after six months of storage, expiry date indicated on the bottle, packaging. The results show that the parameters studied $(\mathrm{pH}$, alcohol content, total titratable acidity and volatile acidity) change during storage time. However, during the six months of storage, these parameters remain within the set limits for beer.
} 

en mairie de Bujumbura, Burundi.

Conclusion and application of results: The taste of beer can actually change during storage time, but beer is considered still in good condition before the six months of storage. However, "this good state" is still different from the state corresponding to the storage time $t=0$. According to these results, the consumer and the beer seller will understand each other.

Keywords: Amstel beer, Amstel bock, physico-chemical parameters, taste, degradation, storage, Bujumbura.

\section{INTRODUCTION}

La conservation des produits alimentaires est le problème majeur que doit résoudre l'industriel alimentaire parce que ce dernier se doit de mettre sur le marché des produits ayant des caractéristiques constantes (Spinnler, 2008). La bière est une boisson désaltérante, consommée dans le monde entier et obtenue à partir de certaines céréales, notamment l'orge, le maïs, le riz et le blé, associées à l'eau et au houblon (Caporale et al., 2004 ; Boivin 2005). Au Burundi, la bière fait partie de la culture burundaise. Par exemple, partager une bière permet de réconcilier certaines parties en conflit (Sommers, 2013). A côté des bières locales comme, la bière de sorgho et la bière de sorghomaïs, les bières Primus, Nyongera et Amstel (Amstel bock et Amstel beer) sont fabriqués par la Brasserie et limonaderie du Burundi (BRARUDI) et consommées par la population burundaise (Fien Van Wolvelaer, 2015). Ainsi, analyser un produit alimentaire qui est consommé à ce rythme est plus que nécessaire. L'étude sur de quelques boissons consommées au Burundi vient d'être effectuée (Ndamanisha et al. 2016 a, Ndamanisha et al. b). Cependant, la dégradation de ces boissons au cours de leur conservation au Burundi n'est encore analysée. L'évolution des caractéristiques gustatives d'une bière varie essentiellement selon les types et donc selon le mode conditionnement. Pour les consommer avec leurs caractéristiques constantes, la plupart des bières sont pourvues d'une date de

\section{MATERIELS ET METHODES}

Trois jours après la sortie d'une caisse contenant 24 bouteilles de la bière Amstel bock et d'une autre contenant 12 bouteilles d'Amstel beer, les paramètres physico-chimiques de ces deux bières ont été mesurés. Les bouteilles non ouvertes ont été conservées dans une salle non climatisée et à l'air libre en Mairie de Bujumbura pendant un mois. Après ce temps de conservation, les mêmes paramètres physico-chimiques ont été mesurés et péremption à cause de la variation de la stabilité de l'arôme et du goût (David et Thibault, 2013). La date d'expiration marquée sur les bouteilles contenant la plupart des bières est de six mois. Cependant, comme le souligne Jolly (2004), les buveurs sont capables de déterminer la variation de son goût. Dans ce cas, les vendeurs et les consommateurs se trouvent dans un état de malentendu. Des fois, les consommateurs disent que le produit vendu n'a plus le goût de la bière et ils pensent que la bière a été dégradée. Les vendeurs quant à eux, montrent la date d'expiration marquée sur la bouteille d'emballage pour montrer que la bière est encore en bon état. Si cette étude a été réalisée en Europe (Commission européenne, 2005), il est temps de vérifier si les caractéristiques de ces bières consommées au Burundi restent constantes avant cette date d'expiration. Ce malentendu sera dans ce cas géré avant sa naissance. Pour contribuer à la gestion de ce malentendu, il est utile de mener une étude sur ces bières consommées. Pour commencer, l'étude va porter sur deux bières qui sont plus consommées à Bujumbura (Wolvelaer, 2015). Dans ce travail, nous allons suivre l'évolution de quelques paramètres physico- chimiques au cours de la conservation des bières Amstel (bock et beer) pendant six mois, date d'expiration marquée sur la bouteille de conservation. La comparaison entre les deux bières va nous montrer la bière la plus stable.

les bouteilles non ouvertes ont encore été conservées pendant un mois. Le même processus de mesure et de conservation a été effectué pendant six mois. La période de six mois a été choisie parce que l'étiquette sur la bouteille d'emballage de la bière (Amstel bock et Amstel beer) montre une durée d'expiration de six mois. La teneur en alcool (TA), du pH, de l'acidité totale titrable (ATT), de l'acidité volatile (AV) et de l'acidité fixe (AF) ont 
été mesurés à l'Université du Burundi en utilisant la méthode de Lonvaud (1995),

Traitement statistique : La comparaison entre deux moyennes expérimentales (de l'Amstel beer et de

\section{RESULTATS et DISCUSSION}

La figure 1 montre les bouteilles d'emballage des bières Amstel beer et Amstel bock. II est facile de noter que les

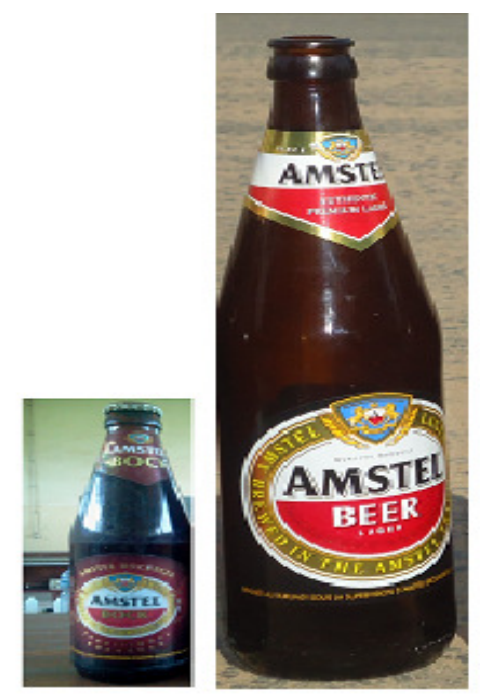

l'Amstel bock) a été faite en utilisant la méthode citée par Skoog et al (2006) et au degré de confiance de $95 \%$.

volumes de ces bouteilles sont différents. Cependant, les dates d'expiration sont les mêmes.

Figure 1 : Bouteilles d'emballages des bières Amstel bock et Amstel beer

Analyse de la variation des paramètres physicochimiques des bières étudiées : Le tableau 1 donne les valeurs de l'évolution des paramètres de la bière Amstel beer et de la bière Amstel bock. On peut remarquer que le TA diminue en fonction du temps. Au cours de sa conservation, une boisson alcoolique subit des transformations dont la transformation de l'éthanol contenu dans la bière en acide acétique (Renouf, 2013). Cependant, on peut noter le taux d'alcool reste dans les deux limites reconnues par les normes générales (Andrews et al. 2013). Aussi, le pH des deux bières diminue au cours de leur conservation. Ce phénomène est facile à expliquer si on le lie à celui du TA. En effet, si l'alcool se transforme en acide, il est clair que le $\mathrm{pH}$ du milieu diminue. Au fur à mesure que l'alcool se transforme, l'acidité, surtout l'acidité volatile augmente et on sait que le pH diminue avec l'acidité du milieu.

Tableau 1 : Valeurs des paramètres des bières Amstel beer et Amstel bock

\begin{tabular}{|l|l|l|l|l|l|l|l|l|}
\hline Paramètres & $\begin{array}{l}\text { pH } \\
\text { Amstel } \\
\text { beer }\end{array}$ & $\begin{array}{l}\text { pH } \\
\text { Amstel } \\
\text { bock }\end{array}$ & $\begin{array}{l}\text { TA } \\
\text { Amstel } \\
\text { beer }\end{array}$ & $\begin{array}{l}\text { TA } \\
\text { Amstel } \\
\text { bock }\end{array}$ & $\begin{array}{l}\text { ATT } \\
\text { Amstel } \\
\text { beer }\end{array}$ & $\begin{array}{l}\text { ATT } \\
\text { Amstel } \\
\text { bock }\end{array}$ & $\begin{array}{l}\text { AV } \\
\text { Amstel } \\
\text { beer }\end{array}$ & $\begin{array}{l}\text { AV } \\
\text { Amstel } \\
\text { bock }\end{array}$ \\
\hline Temps & 4,70 & 4,42 & 5,15 & 6,03 & 80,01 & 80,12 & 16,37 & 15,15 \\
\hline 0 & 4,60 & 4,36 & 5,02 & 6,00 & 81,06 & 81,06 & 16,73 & 15,37 \\
\hline 2 & & 4,30 & & 5,85 & & 83,29 & & 15,74 \\
\hline 3 & 4,15 & 4,20 & 4,80 & 5,81 & 83,67 & 83,45 & 17,49 & 15,77 \\
\hline 4 & & 4,12 & & 5,78 & & 85,6 & & 16,18 \\
\hline 5 & 3,96 & 4,09 & 4,46 & 5,7 & 85,40 & 86,78 & 18,03 & 16,39 \\
\hline 6 & 3,80 & 4,05 & 4,10 & 5,45 & 86,06 & 88,07 & 18,17 & 16,65 \\
\hline
\end{tabular}



en mairie de Bujumbura, Burundi.

Néanmoins, on constate que pendant les six mois, date d'expiration indiquée, le $\mathrm{pH}$ reste dans les normes établies par la théorie de la bière. Ces normes varient entre 3,5 et 7 (Andrews et al. 2013). Par contre, l'ATT augmente en fonction du temps de conservation des bières étudiées. Ce phénomène renforce l'explication donnée aux phénomènes précédents (diminution du TA et du pH). En s'oxydant, l'éthanol $\left(\mathrm{CH}_{3} \mathrm{CH}_{2} \mathrm{OH}\right)$ se transforme en acide acétique $\left(\mathrm{CH}_{3} \mathrm{COOH}\right)$ qui est un acide volatile, auquel s'ajoutent les acides fixes (tartrique, malique) contenus dans la bière. II est alors clair que la quantité d'ATT, produit de la réaction augmente en fonction du temps de la réaction. Aussi, les valeurs de l'ATT sont dans les limites reconnues par la théorie des bières. Ces limites sont de 80,4 et 142,858 méq/l. (Andrews et al. 2013). Le tableau 1 montre que l'AV augmente au cours du temps de la conservation des bières. L'acidité volatile étant une partie de l'ATT, il est facile de noter que ce phénomène s'explique (comme le cas précédent) par la transformation de l'éthanol en acide acétique. Aussi, l'acide tartrique $(\mathrm{HOOC}-\mathrm{CHOH}-\mathrm{CHOH}-$ $\mathrm{COOH}$ ) donne l'acide acétique sous l'influence des bactéries du genre lactobacilles (Hervé et al. 2008). Comme les autres, ce paramètre reste dans les limites établies par la théorie des bières.

Comparaison de la stabilité de la bière Amstel bock et Amstel beer : Une bière plus stable va rester avec un pur franc et net (Latour A.1999). C'est pourquoi il faut faire une comparaison de la stabilité des deux bières étudiées. Pour étudier la comparaison de l'évolution des paramètres physico-chimiques des deux bières, la méthode graphique est la mieux indiquée. Avec un écarttype de 0,07 , les deux moyennes des pentes des deux courbes de variation des paramètres physico-chimiques mesurés ont été comparées au degré de confiance de $95 \%$.

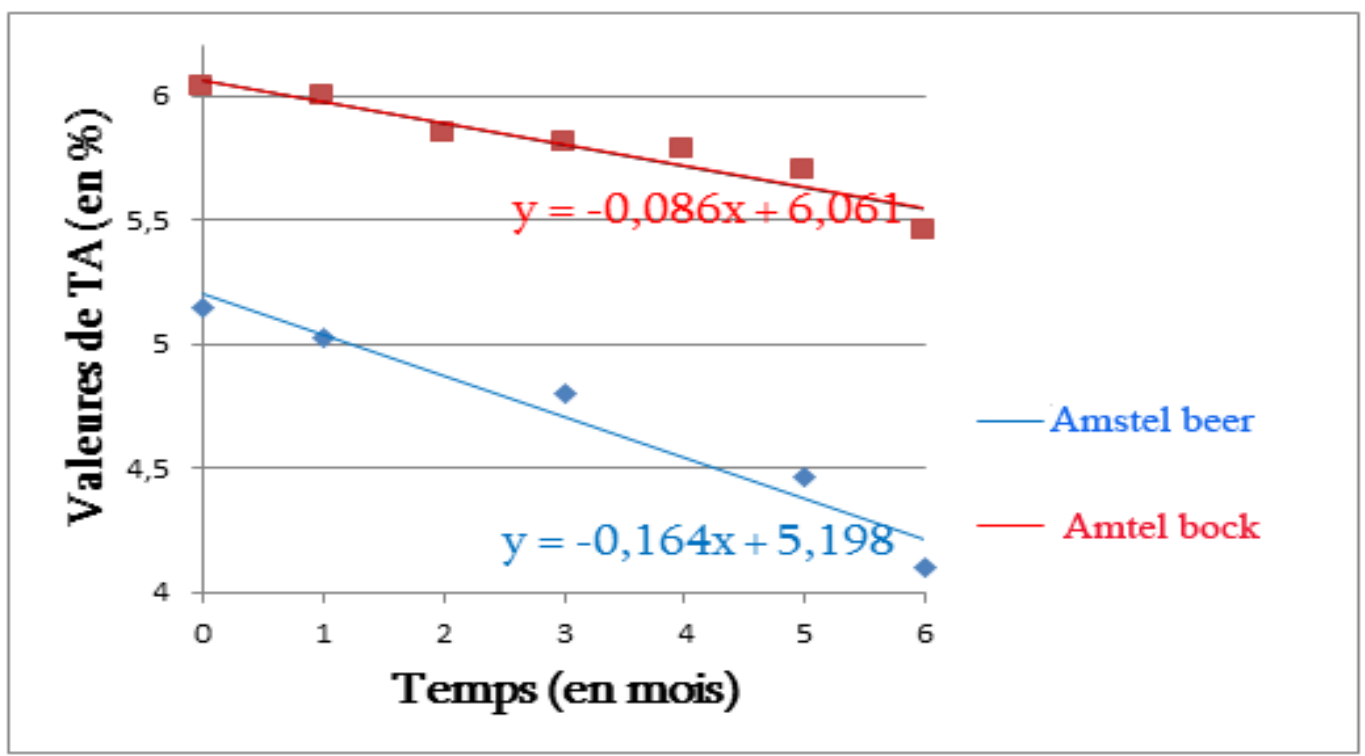

Figure 2 : Évolution du TA des bières Amstel beer et Amstel bock

La figure 2 indique l'évolution du TA des deux bières. L'évolution du taux d'alcool de la bière Amstel beer est une droite d'équation $y=-0,164 x+5,198$, tandis que l'équation de la courbe du TA de la bière Amstel bock est $y=-0,086 x+6,061$. A partir de ces deux équations, on voit que les deux droites ont une pente négative, ce qui confirme que le TA diminue au cours du temps de conservation. La comparaison des valeurs absolues des pentes des deux droites montre que la différence expérimentale est de 0,078 et que la valeur calculée au degré de confiance de $95 \%$ est de 0,89 . Ce qui indique que les deux pentes sont significativement différentes. Par conséquent, le TA de la bière Amstel beer diminue plus rapidement que celui de la bière Amstel bock. On peut penser que la transformation de la bière Amstel bock est plus lente que la bière Amstel beer. La Figure 3 indique l'évolution du pH de la bière Amstel beer et la bière Amstel bock. 


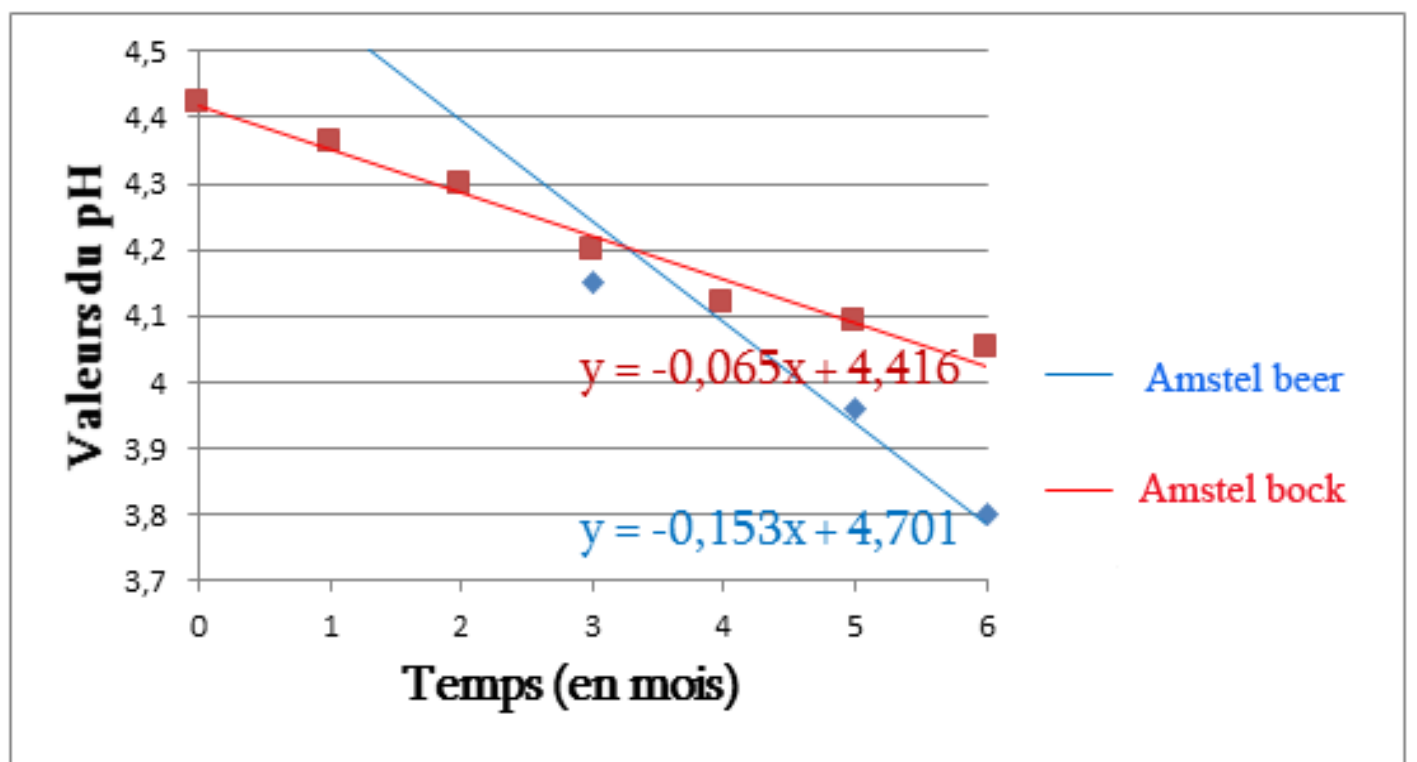

Figure 3 : Évolution du pH des bières Amstel beer et Amstel bock

La courbe de l'évolution du pH de la bière Amstel beer est une droite d'équation $\mathrm{y}=-0,153 \mathrm{x}+4,701$, tandis la courbe $\mathrm{du} \mathrm{pH}$ de la bière Amstel bock a une droite d'équation $\mathrm{y}=$ $-0,065 x+4,416$. Les deux pentes étant négatives, le $\mathrm{pH}$ des deux bières étudiées diminuent en fonction du temps comme nous l'avons dit précédemment. Cependant, on remarque que le $\mathrm{pH}$ de la bière Amstel beer diminue plus rapidement que celui de la bière Amstel bock. En effet, si on compare la pente des deux droites avec le même traitement statistique, on remarque que la pente de la droite correspondant à la bière Amstel beer $(0,153)$ est beaucoup plus grande que celle de la bière Amstel bock $(0,065)$. La diminution du $\mathrm{pH}$ provenant de la transformation de la bière en acide, on peut penser que la transformation de la bière Amstel bock est plus lente que la bière Amstel beer. La raison est que la bière Amstel bock, au temps $\mathrm{t}=0$, est plus acide $(\mathrm{pH}=4,42)$ que la bière Amstel beer $(\mathrm{pH}=4,70)$; ce qui diminue un peu la transformation en acide (déplacement de l'équilibre). La figure 4 montre l'évolution de l'ATT de la bière Amstel beer et la bière Amstel bock.

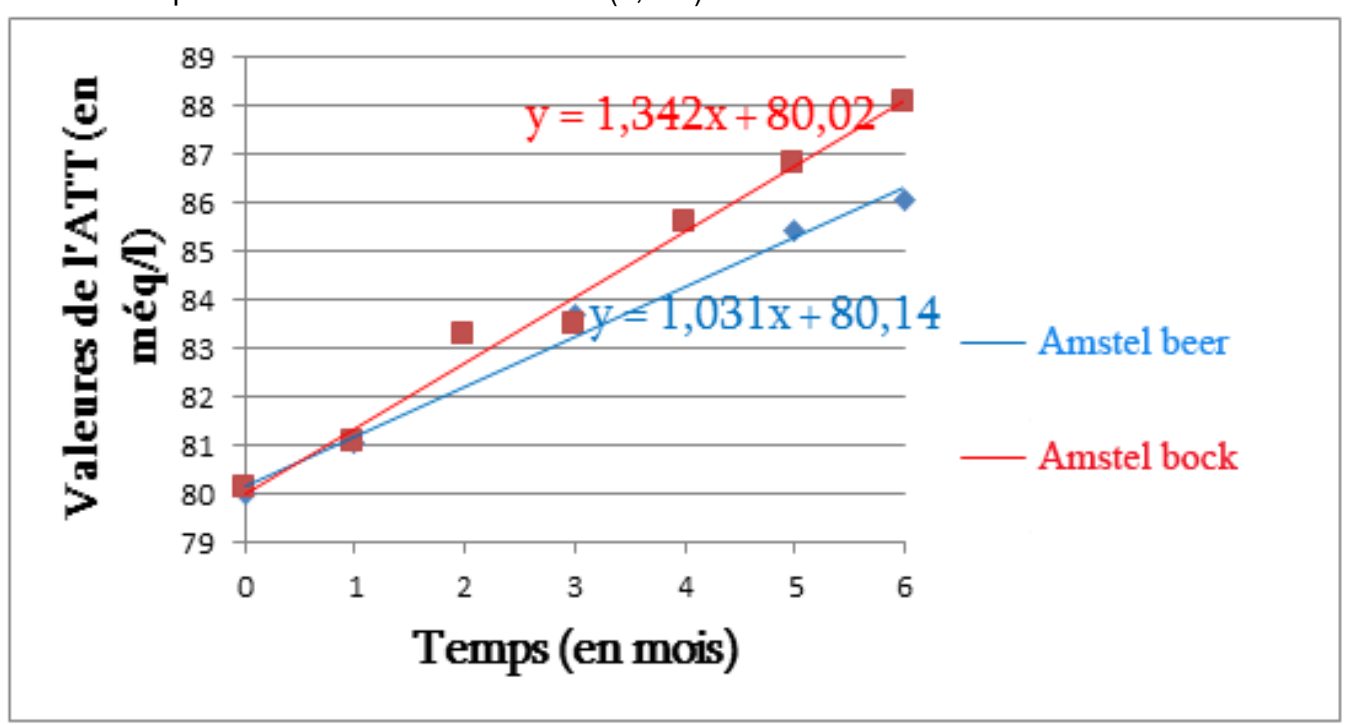

Figure 4 : Évolution d'ATT des bières Amstel beer et Amstel bock 
L'équation de la droite de la courbe de l'évolution de l'ATT de la bière Amstel beer est $y=1,031 x+80,14$ alors que celle de la courbe d'ATT de la bière Amstel bock est $y=1,342 x+80,02$. Contrairement aux tendances linéaires des TA et des $\mathrm{pH}$, les pentes de ces droites sont positives. Dans ce cas, I'ATT des deux bières augmente en fonction du temps de conservation. Cependant, comme indiqué plus haut, l'ATT de la bière Amstel bock augmente plus rapidement que celui de la bière Amstel beer. En effet, la comparaison des pentes des deux droites indique que la pente de la droite correspondant à la bière Amstel bock $(1,342)$ est plus grande que celle de la bière Amstel beer $(1,031)$. L'augmentation de l'ATT provient de la transformation de l'alcool de la bière en acide. Ceci indique que la transformation de l'alcool de bière Amstel bock est plus rapide que celle de la bière Amstel beer. La raison est que la bière Amstel bock, au temps $t=0$, contient beaucoup d'alcool $(6,03 \%)$ que la bière Amstel beer $(5,15 \%)$. La figure 5 montre l'évolution de l'AV de la bière Amstel beer et la bière Amstel bock. Les équations de la droite de la courbe de l'évolution de l'AV de la bière Amstel beer et celle de la droite de la courbe d'AV sont respectivement $y=0,307 x+16,43$ et $y=0,249 x+15,14$ Comme pour le cas de l'ATT, il est claires que l'AV diminue en fonction du temps de conservation parce ces pentes sont positives.

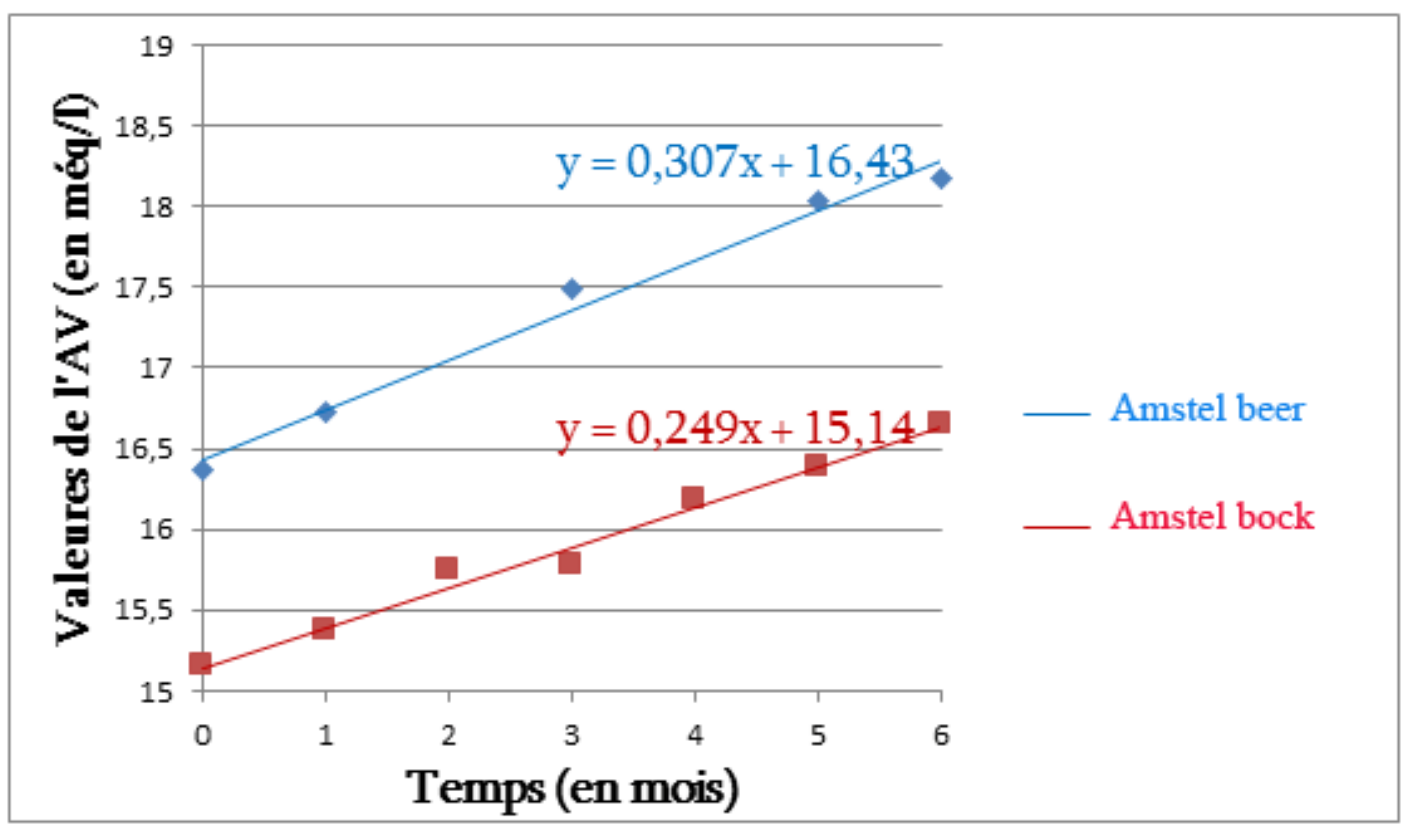

Figure 5 : Évolution d'AV des bières Amstel beer et Amstel bock

II est aussi facile de remarquer que la pente de la droite correspondant à la bière Amstel beer $(0,307)$ est plus grande que celle de la bière Amstel bock $(0,249)$. L'augmentation de l'AV est due à la transformation de l'alcool en acide acétique. Ceci indique que, on peut penser que la dégradation de la bière Amstel bock est plus lente que celle de la bière Amstel beer. L'alcool étant un désinfectant, on peut penser que les bactéries causant

\section{CONCLUSION.}

L'objectif de ce travail était de vérifier que les deux bières consommées au Burundi (Amstel beer et Amstel bock) ne se dégradent pas pendant leur conservation en Mairie de Bujumbura. Pour ce faire, l'évolution des paramètres la dégradation de la bière se développent lentement dans la bouteille de l'Amstel bock. En analysant l'évolution des paramètres physico-chimiques des deux bières, toutes conservées dans les mêmes conditions et dans le même milieu : la mairie de Bujumbura, il est possible d'affirmer que la bière Amstel beer se dégrade plus rapidement que la bière Amstel bock.

physico-chimiques de ces bières, à savoir : la teneur en alcool (TA), le pH, l'acidité totale titrable (ATT), l'acidité volatile $(\mathrm{AV})$ a été suivie pendant les six mois de conservation. Cette période a été choisie parce que la 
durée d'expiration proposée par le producteur est de six mois. Les résultats ont montré que les paramètres physico-chimiques de la bière Amstel bock varient en fonction du temps de conservation. Cependant, pendant les six mois de conservation, ces paramètres restent dans les limites établies par la théorie des bières. Ceci indique que, d'une part le goût de la bière peut varier en fonction du temps de conservation et d'une autre part, la bière n'est pas encore abimée avant les six mois de conservation. Ainsi, le goût d'une bière conservée

\section{REFERENCES}

Andrews et al. 2013. Études des modifications proposées à la norme d'identité de la bière du canada, Bière Canada $p 22$.

Boivin C., 2005. La bière : son histoire, sa fabrication et sa dégustation. Lac Beauport, Québec, Canada : Éditions Arion.

Caporale G. et Monteleone E., 2004. Influence of information about manufacturing process on beer acceptability. Food Qual. Preference, 15 (3), 271-278.

Commission européenne, 2005. Les attitudes des consommateurs à l'égard de l'étiquage, Optem $p$ 39.

David L. G. et Thibault M. 2013. Les saveurs gastronomiques de la bière, Tome II, p 164 Editions Druide.

Hervé A., et al. 2008. Les bactéries lactiques en œnologie, Lavoisier /Tec \& doc, $172 p$.

Jolly E. 2004. Boire avec esprit. Bière de mil et société dogon, Société d'Ethnologie, Nanterre, 499 p.

Latour A. 1999. Les brasseurs et la bière, éditions Créer, $64 \mathrm{p}$.

Lonvaud A., Rivoal F., Vidal M. 1995. Analyse du vin. Bulletin de l'Union des Physiciens, Vol 89 : 1173-1187.

Renouf V. 2013. Les fermentations malolactiques dans les vins. Mécanismes et applications pratiques, Lavoisier/Tec \&Doc, 223 p.

Ndamanisha J. C. and Nkuririmana J. 2016. Effect of local banana wine consumption on uric acid elimination: a quantitative analysis, African Journal of science and Research. 5: 7-9.

Ndamanisha J.C. Ndayihimbaze V. 2016. Effects of water consumption on uric acid elimination: elimination of stocked uric acid by beer consumption, African Journal of Science and Research 5: 74-77.

Sommers M. 2013. Adolescents and Violence. Lessons from Burundi. Retrieved from Institute of pendant un mois n'est pas le même que celui de la même bière conservée pendant six mois. On peut donc conclure que le consommateur peut avoir raison lorsqu'il dit que telle bière n'a pas le goût d'une bière qu'il consommait avant. Cependant, ceci n'indique pas que le vendeur a tort s'il montre que la bière n'est pas encore abimée avant la date d'expiration. Aussi, il a été montré que la bière Amstel bock est plus stable que la bière Amstel beer. Le changement de goût dans cette dernière bière est plus rapide que dans l'Amstel bock.

Development Policy and Management \& University of Antwerp http://www.ua.ac.be/iob.

Skoog D.A., West D.M. et Holler F.J. 2006. Chimie analytique, de boeck, Bruxelles, $870 \mathrm{p}$.

Spinnler H. E. 2008 Transformation et conservation des produits agroalimentaires, Techniques de l'ingénieur, F3450, 1-2.

Wolvelaer F.V. 2015, Alcohol addiction treatment programs in Bujumbura, Burundi : A qualitative analysis towards the relationship between local perceptions of alcohol use and ideas about treatment strategies, Thèse de Master, Université de Gant. 